\title{
Digital transformation: why companies resist what they need for sustained performance
}

\author{
Johan Magnusson, Viktor Elliot and Johan Hagberg
}

Johan Magnusson is based at the Swedish Center for

Digital Innovation,

Department of Applied IT, University of Gothenburg, Gothenburg, Sweden and Kristiania University College, Oslo, Norway. Viktor Elliot and

Johan Hagberg are both based at the Department of Business Administration, University of Gothenburg, Gothenburg, Sweden.
(C) Johan Magnusson, Viktor Elliot and Johan Hagberg. Published by Emerald Publishing Limited. This article is published under the Creative Commons Attribution (CC BY 4.0) licence. Anyone may reproduce, distribute, translate and create derivative works of this article (for both commercial and non-commercial purposes), subject to full attribution to the original publication and authors. The full terms of this licence may be seen at http:// creativecommons.org/licences/ by/4.0/legalcode

\section{When everybody is doing it}

Many have envisioned digital transformation as a way to dramatically transform industry and society. Firms seem to compete in voicing accolades of significant opportunity, yet at the same time, a burgeoning body of literature has considered why significant transformation seems to lag.

Digital transformation can be understood both as a societal phenomenon and as a method for business development. We focus on the latter, the utilization of digital technologies by organizations in the establishment of new, digital value streams or how organizations use digital to transform from existing business models to newer ones.

In this field, research has focused on the increasing role of digital as a strategy, whether in itself or as an integral part of the broader business strategy. As noted by Bharadwaj et al. (2013), from early attempts to treat digital strategy as something separate, approaches are increasingly shifting to what researchers refer to as a "digital business strategy," a fusion of the digital and the traditional. This is elaborated by Tekic and Koroteev (2019) in their identification of four generic digital transformation strategies. These range from the "disruptive," meaning the substantive change of a business model, to the "proud to be analog" strategies, which use the functional separation of new digital business from traditional business.

There are various paths organizations can take on their journey to digital. Early contributions identified the journey from "beginners," taking the first unsteady steps with low digital intensity and low levels of transformation, to the "digirati," demonstrating high digital intensity and high levels of transformation. In regard to the level of digital intensity, studies have shown that this is industry specific, where industries such as finance, telecommunications and software have been digitally intense since the 1990s compared to utilities and construction. The choice of path to digital transformation seems to be contingent on the industry's existing dependence on digital technologies. But there are few industries that can presently be considered exempt from the requirements of digital transformation.

Despite the clear hyperbole and suggestiveness of digital transformation, several studies have identified significant shortcomings in the way organizations approach digital transformation. As digital transformation is cumbersome and associated with risk (as is any change in an organization's value streams), it is only natural that organizations and their employees should develop and use strategies to counteract transformation. Previous research has identified strategies associated with defense and coping as core in the 
resistance to transformation. In line with this, we explore how organizations engage in resistance and what strategies they use to avoid digital transformation. To fuel our exploration, we use illustrations from our own studies of digital transformation in multiple industries. The rationale in addressing these strategies is to contribute to management insight into how an organization can succeed in digital transformation and ensure its longterm performance in an increasingly digital economy.

\section{Illusive option of strategy zero}

"Strategy zero" refers to an established organization's original strategy that is continuing along the existing path. In our context, it involves the organization avoiding digital transformation, often at the cost of missing the opportunities digital transformation.

From previous studies, we know that digital transformation in established firms is counteracted by their established routines, inertia and path dependencies. Incumbent firms have an established, installed and fine-tuned base that enables them to continue along their existing trajectory. They also have fixed revenue streams and an antipathy to cannibalization and other forms of behavior that may harm their existing business. They are equipped with an active "immune system" that shields them from radical shifts.

History is full of cautionary tales of organizations that have failed to correctly assess the trajectory of technology and chosen to follow strategy zero. From Kodak to Blockbuster to Nokia, these examples teach us that the case for steering away from strategy zero is compelling.

\section{Strategies for digital decoupling}

In this study, we identify the phenomenon of digital decoupling with respect to how established organizations avoid digital transformation. In line with Meyer and Rowan (1977), we define digital decoupling as a strategy that allows for a misalignment of the formal and the informal and opens up for the parallel handling of the digital (new business) and nondigital (existing business). We see this approach enacted in organizations that, for example, spin out their digital ventures instead of integrating them or that create chief digital officer positions without mandate. We also see digital decoupling in the manner in which an organization interacts with external actors, such as consultants or researchers tasked with studying digital transformation. It is via our exploration of such digital decoupling in interactions with external parties that we make our contribution.

Previous research on decoupling is vast and multifaceted, with some findings pointing to the purposefulness of decoupling and others indicating its negative implications for sustainable success. As noted by Brignall and Modell (2000), decoupling can also be perceived as a means through which ambiguous and contradictory goals are balanced $-\mathrm{a}$ perspective that illustrates the innate functionality of decoupling.

In this paper, we delineate four strategies of digital decoupling. We present each strategy in relation to the level where it is used (organizational or individual), and whether it is an act of defense or coping. The strategies are described and illustrated in the main sectors of Figure 1.

\section{"There are few industries that can presently be considered} exempt from the requirements of digital transformation." 


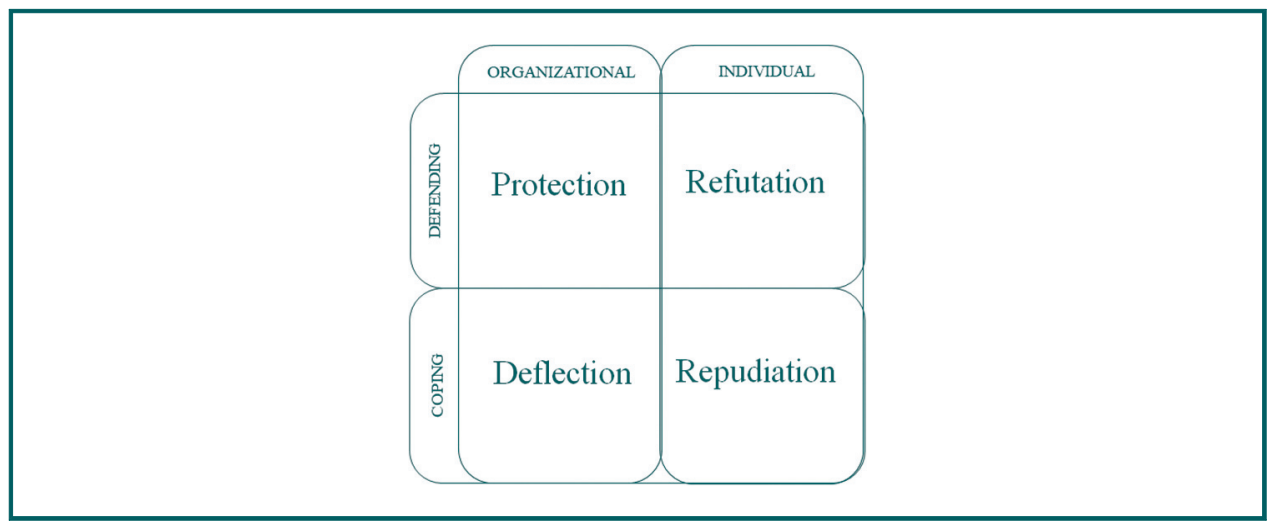

We chose one occurrence to illustrate each strategy, described below, but each strategy has been observed on multiple occasions and in multiple forms by the research team.

\subsection{Strategy 1: protection}

One of the authors was invited to give a board-level keynote speech on how increased access to data leads to new value-creating opportunities (Tsvetkov and Chekanov, 2019) and the demands such access in turn places on an organization venturing into the datadriven business-scape. The speaker based this presentation on a research project that had rendered some interesting results on the relatively low adoption level of data-driven practice in one specific industry, as well as in the organization requesting the speech.

The organizers asked the researcher to share the presentation ahead of the seminar. The sharing was met with questions and suggestions for how the material could be improved for the specific audience, mostly in the form of suggestions for clarifications and repeated statements that the audience was not ready to be challenged. The organizers requested that, as they saw it, the damning tone in the presentation toward the industry and firm should be toned down, so as not to make the listeners "feel too bad about the firms' initiatives." This was met with resistance from the researcher and increasing tension with the organizers throughout the email correspondence. Right before the seminar, the organizers reached out again, in essence summarizing their feedback into, "Whatever you do, don't challenge them [the board]."

As the example shows, this organization attempted to shield and protect its members from potentially damning observations from the outside. An external assessment indicated that digital transformation was not yet completed and that the organization had significant hurdles to overcome; the organization deemed this assessment a threat and viewed the findings as needing to be counteracted. Thus, protection strategies can be considered as various forms of filtering and editing, in which organizations shy away from potential shortcomings in existing practice and digital transformation initiatives, with the intent of safeguarding their identity as leading firms.

\subsection{Strategy 2: refutation}

As part of a funding application procedure for a larger research program, a research team was invited to give an oral presentation of their application before an evaluation committee. The suggested program focused on the digital transformation of one specific industry. Until then, digitalization within that industry was associated with a smaller number of new entrants and considered a niche phenomenon. The researchers provided a number of examples of how the industry in question would come to face all-embracing changes because of 


\section{"Digital transformation in established firms is counteracted by their established routines, inertia, and path-dependencies."}

digitalization, including industry offerings, consumption behaviors and most aspects of the companies' operations in various ways. The presentation was followed by questions and comments from the committee. One of the committee members, a senior manager with previous industry experience, was not at all convinced about the portrayal of the future and ironically suggested that the research program, if granted, should include a work package on digital potatoes. This served as an argument that the industry in question was handling physical products and therefore would not be particularly affected by digital transformation. In the final decision - in which the application was rejected - one of the motives was that the committee considered digitalization a narrow topic that would continue to have limited impact on the industry.

This vignette exemplifies individuals acting to safeguard the current state of affairs. By refuting the message about changes within the industry, the individual tries to adjust the message so that it fits an alternative depiction in which the industry will remain largely unaffected. Through this act of defense, one may continue acting as before, avoiding unwanted appeals to adjust. Thus, strategies of refutation include efforts to argue against, deny or ignore depictions of digital transformation.

\subsection{Strategy 3: deflection}

In this example, a group of individuals with exemplary, relevant capabilities led a research project designed to use artificial intelligence functionality to assess new data utilization methods and the shift toward dynamic pricing. The initial dialogue with one of the partner firms was positive, where the organization saw the immense, game-changing potential of the approach and the opportunity costs associated with several international industry leaders recently having started to move in this direction. The research team was promised excellent access to the data necessary for this type of analysis, and legal aspects associated with data protection were addressed immediately.

Upon starting the project, the initial promises swiftly grew bleaker. To access the data, the researchers were asked to secure the cooperation of the decentralized offices that housed their own data. This proved cumbersome, as it was not given adequate central support within the organization. With a growing sense of unease, the researchers were in continuous contact with the data owners, trying to get them to buy into the idea and to provide access to the data. The researchers even offered to work with the data on-site, under the direct supervision of the information technology (IT) staff of each office. The organization continuously repeated the importance of the project and their willingness to actively contribute to it, but gradually their support turned from allowing data sharing to supporting the collection of external data. In one of the final meetings with the data stewards of the organization, the stewards stated, "We could probably share the data, but have you checked elsewhere?"

Although it is quite common for access to various sources of company data to change over the course of a research project, this case illustrates a deflection of the responsibility for the digital transformation of the organization. In particular, the organization persistently maintained their interest in the research and the instrumental position of the research team in finding new ways of using the existing data for new analyses. Still, the organization saw the data release as threatening and coped with this by conceding that the researchers should get access, but only as a very last resort. This form of digital window-dressing is quite common, and Nell et al.'s (2020) survey testified to the allure of embarking on ambitious IT projects that nonetheless offer slim chances of leading to actual digital 
transformation. Thus, deflection strategies emphasize an expressed ambition and commitment to digital transformation that is portrayed to external stakeholders but are not reflective of what is really going on within the organization.

\subsection{Strategy 4: repudiation}

In late October of 2020, the chief executive officer of a large corporation gave a keynote speech at an industry conference about the hype surrounding technological innovators within the industry. In the speech, he downplayed the disruptive nature of these innovators, stating that they are, although interesting as a phenomenon, not a relevant threat to the established actors in the industry [See Pisoni (2020) for a tangent on this specific industry].

This speech, and the message that it conveyed, resonated with several earlier experiences from the same industry, including an executive responding to our analysis of a similar actor's loss of revenue because of innovator firms squeezing them in terms of profit shares. The executive nonetheless maintained, "But that doesn't say anything about us." At the same conference, another executive disregarded the transferability of an assessment of a competitors' strategic choice (which was similar to their own) by stating, "Of course this doesn't mean that [...]" We were continuously met with wavering attempts to deny the validity of an approach that in essence could challenge the organizations' own actions and call into question the appropriateness of their strategies.

This vignette illustrates the repudiation of facts, findings and phenomena as not being valid for a particular organization's digital transformation. Instead of accepting the claims and being open to potential transferability, the individual in question debunks the claim and closes the door to new insights that may prove valuable for their digital transformation. We found that this particularly holds true for risks that are high and previously unheard of, such as disruption from new entrants into the market. Other examples of repudiation include Schmid's (2019) exposé of a Western European mobility provider, which detailed thoughtprovoking examples such as employees locking away smartphones and innovating around newly introduced system solutions. Thus, repudiation strategies include an individual's dismissal of new technologies for their daily work, as well as a broader ignorance of the risk of disruption as something unrelated to that specific individual.

\section{Strategies of digital recoupling}

In working with organizations in several industries for the past several years, we have also observed examples of how firms succeed with digital transformation. With the identified strategies for digital decoupling, we see that successful firms use counter-strategies for digital decoupling; that is, strategies to ensure that transformation is not avoided. We refer to these strategies as digital recoupling.

In regard to how these firms handle the influx of external influences, they show traits of what the literature has referred to as integrative capabilities - that is, dynamic capabilities related to creating positive interaction among disparate resources. As noted by Wimelius et al. (2021), integrative capabilities allow the organization to orchestrate networked innovation

\section{"From Kodak to Blockbuster to Nokia, these examples teach us that the case for steering away from strategy zero is compelling."}


and, as such. become key in safeguarding the influx of external influences. We present the identified strategies for digital recoupling in order below (Figure 2).

Openness counteracts protection; in that the organization avoids taking a defensive stance against external insights that may be difficult to hear. By staying open to externally generated insights, the organization can counteract the loss of information that may prove critical for future operations. In some organizations we have worked with, this has been implemented through formal means such as external advisory boards (with representatives from both academia and industry); in other organizations, openness is part of the very culture, including practices such as seminars with external parties that frequently reflect on issues of potential value to the organization.

Reflectiveness on the individual level counteracts refutation, with the individual regularly reflecting on the soundness of existing operations. Some organizations enact reflectiveness through team members taking on the role of devil's advocate, explicitly challenging the status quo and the current set of initiatives. This form of spirit aspiring to continuously challenge the status quo requires nurture and empowerment, which is why organizational culture and values become essential (Lichtenthaler, 2019).

Proactiveness, which counteracts deflection at the organization level, refers to coping with disruptive changes in the environment through flexibility and a swift manner of addressing issues. Organizations that struggle to sense, seize and reconfigure new options can be proactive by decreasing the thresholds of corporate buy-in for new initiatives (see the example from Amazon's prioritization process). Simplifying the bureaucracy for gaining access to data, speeding up the process of onboarding new partners that could assist and creating sandbox environments for digital development are examples of successful manifestations of this counter-strategy that we have seen in our studies.

Committedness counteracts repudiation on the individual level. It involves a shared responsibility that includes all individuals in the organization being committed to the organization's digital transformation, instead of resisting it. Organizations that continually restructure their staff offer an example of how this strategy may be implemented by formal means although it may also be enacted through cross-functional teams (Hirsch, 2020).

As these four strategies show, digital recoupling opens the organization to new insights and challenging viewpoints and, as such, signals a more reflective culture in an organization. With digital becoming increasingly pervasive over time, we believe that attempts at digital decoupling are destined to fail in the long run. Instead of treating digital as something that is separate and apart, as noted by Tekic and Koroteev (2019) in their "proud to be analog" strategy, organizations should aspire to find a means through which digital may be

Figure 2 Strategies for digital recoupling

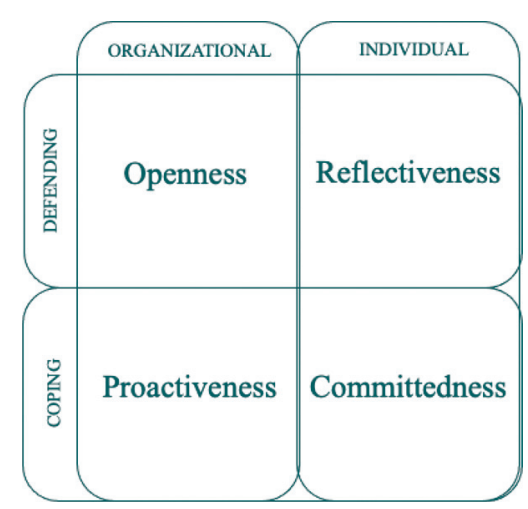




\section{“Organizations shy away from potential shortcomings in existing practice and digital transformation initiatives, with the intent of safeguarding their identity as leading firms."}

Keywords:

Strategy,

Organizational change,

Digital transformation,

Organizational resistance,

Digital decoupling,

Digital recoupling integrated and fused with the existing business model of the organization, that is, recoupled. As argued by Magnusson et al. (2020), this would call for increased pluralism in the manner in which digital transformation is managed and organized. We should accept the innate difference in the digital and aspire for integration rather than decoupling. We hope that the strategies for digital decoupling presented in this paper will provide a cautionary example for managers striving for digital transformation, and that the strategies for digital recoupling may be used as inspiration for a new, more fruitful approach.

\section{References}

Bharadwaj, A., El Sawy, O.A., Pavlou, P.A. and Venkatraman, N. (2013), "Digital business strategy: toward a next generation of insights", MIS Quarterly, Vol. 37 No. 2, pp. 471-482.

Brignall, S. and Modell, S. (2000), "An institutional perspective on performance measurement and management in the 'new public sector'", Management Accounting Research, Vol. 11 No. 3, pp. 281-306.

Hirsch, P.B. (2020), "The sorcerer's apprentice”, Journal of Business Strategy, Vol. 42 No. 1, pp. 77-80.

Lichtenthaler, U. (2019), "Extremes of acceptance: employee attitudes toward artificial intelligence", Journal of Business Strategy, Vol. 41 No. 5, pp. 39-45.

Magnusson, J., Koutsikouri, D. and Päivärinta, T. (2020), "Efficiency creep and shadow innovation: enacting ambidextrous IT governance in the public sector", European Journal of Information Systems, Vol. 29 No. 4, pp. 329-349.

Meyer, J.W. and Rowan, B. (1977), "Institutionalized organizations: formal structure as myth and ceremony", American Journal of Sociology, Vol. 83 No. 2, pp. 340-363.

Nell, P.C., Foss, N.J., Klein, P.G. and Schmitt, J. (2020), "Avoiding digitalization traps: tools for top managers", Business Horizons, in press.

Pisoni, G. (2020), "Going digital: case study of an Italian insurance company", Journal of Business Strategy, Vol. 42 No. 2.

Schmid, A.M. (2019), "Beyond resistance: toward a multilevel perspective on socio-technical inertia in digital transformation", Proceedings of the 27th European Conference on Information Systems (ECIS), Stockholm \& Uppsala, June 8-14, 2019, available at: https://aisel.aisnet.org/ecis2019_rp/105

Tekic, Z. and Koroteev, D. (2019), "From disruptively digital to proudly analog: a holistic typology of digital transformation strategies”, Business Horizons, Vol. 62 No. 6, pp. 683-693.

Tsvetkov, N. and Chekanov, A. (2019), "The data dilemma: how availability can threaten the competitive advantage of data-based firms", Journal of Business Strategy.

Wimelius, H., Mathiassen, L., Holmström, J. and Keil, M. (2021), "A paradoxical perspective on technology renewal in digital transformation", Information Systems Journal, Vol. 31 No. 1, in press.

\section{Corresponding author}

Johan Magnusson can be contacted at: johan.magnusson@gu.se

For instructions on how to order reprints of this article, please visit our website: www.emeraldgrouppublishing.com/licensing/reprints.htm

Or contact us for further details: permissions@emeraldinsight.com 